\title{
The combined effect of Parathyroid hormone (1-34) and whole-body Vibration exercise in the treatment of OSteoporosis (PaVOS)- study protocol for a randomized controlled trial
}

Ditte Beck Jepsen ${ }^{1,2^{*}}$, Jesper Ryg ${ }^{1,2}$, Niklas Rye Jørgensen ${ }^{3,4}$, Stinus Hansen ${ }^{2,5}$ and Tahir Masud ${ }^{1,2,6}$

\begin{abstract}
Background: PaVOS is a randomized controlled trial (RCT) which aims to address the use of whole-body vibration exercise (WBV) in combination with parathyroid hormone 1-34 fragment teriparatide (PTH 1-34) treatment in patients with osteoporosis. PTH 1-34 is an effective but expensive anabolic treatment for osteoporosis. WBV has been found to stimulate muscle and bone growth. Animal studies have shown a beneficial effect on bone when combining PTH 1-34 with mechanical loading. A combined treatment with PTH 1-34 and WBV may potentially have beneficial effects on bone and muscles, and reduce fracture risk.

Methods/design: PaVOS is a multicenter, assessor-blinded, superiority, two-armed randomized controlled trial (RCT). Postmenopausal women ( $n=40$, aged 50 years and older) starting taking PTH 1-34 from outpatient clinics will be randomized and assigned to a PTH 1-34 + WBV-exercise group (intervention group), or a PTH 1-34-alone group (control group).

The intervention group will undergo WBV three sessions a week (12 min each, including 1:1 ratio of exercise: rest, 30 $\mathrm{Hz}, 1 \mathrm{~mm}$ amplitude) for a 12-month intervention period. Both the intervention and the control group will receive PTH 1-34 treatment (20 $\mu \mathrm{g}$ s.c. daily) for 24 months. After 12 months the WBV group will be re-randomized to stop or continue WBV for an additional 12 months.

The primary endpoint, bone mineral density (BMD), will be measured by dual-energy $x$-ray absorptiometry of the total hip and the lumbar spine.

Secondary endpoints, bone microarchitecture and estimated bone strength, will be assessed using high-resolution peripheral quantitative computed tomography (HR-pQCT) of the radius and tibia. Serum bone turnover markers (carboxy-terminal collagen crosslinks (CTX), amino-terminal propeptide of type-l collagen (P1NP), and sclerostin) and functional biomarkers (Timed Up and Go (TUG), Short Physical Performance Battery (SPPB), grip strength, and leg extension power) will be measured to assess the effect on bone turnover, muscle strength, balance, and functionality. Quality of life (EQ-5D), physical activity (IPAQ) and fear of falling (FES-I) will be assessed by questionnaires. Data on adherence and falls incidence will be collected.

(Continued on next page)
\end{abstract}

\footnotetext{
* Correspondence: Ditte.Beck.Jepsen2@rsyd.dk

1 Department of Geriatric Medicine, Odense University Hospital, Odense, Denmark

${ }^{2}$ Institute of Clinical Research, Faculty of Health Science, University of

Southern Denmark, Odense, Denmark

Full list of author information is available at the end of the article
} 
(Continued from previous page)

Discussion: The PaVOS study will investigate the effects of WBV in combination with PTH 1-34 on bone parameters in postmenopausal women.

Trial registration: ClinicalTrials.gov, ID: NCT02563353. Registered on 30 September 2015.

Keywords: Osteoporosis, Whole-body vibration, Parathyroid hormone, Teriparatide, Bone mineral density, Bone quality, RCT

\section{Background Introduction}

Osteoporosis is defined by the World Health Organization (WHO) as a disease with low bone mass and impaired microarchitecture leading to a high risk of fragility fractures [1].

Fragility fractures of the hip are associated with significant morbidity, institutionalization, and a 1-year mortality of $>20 \%$ [2]. Vertebral fractures are also associated with osteoporosis and can lead to significant pain, disability, morbidity, and reduced quality of life [3]. Osteoporotic fractures cause a significant disease burden in developed countries [4] and a recent study of the societal burden imposed by osteoporotic fractures in Denmark showed an estimated yearly cost of $€ 1.563$ billion [5].

The parathyroid hormone 1-34 fragment teriparatide (PTH 1-34) is the most commonly used anabolic agent for the treatment of osteoporosis. The Fracture Prevention Trial showed that PTH 1-34 significantly reduced the incidences of vertebral and non-vertebral fractures [6]. However, the relatively high cost of PTH 1-34 $(€ 7103$ per year) restricts its use in osteoporotic patients to those with the highest fracture risk or, those who had inadequate response to, or cannot tolerate more commonly used anti-osteoporotic agents, such as bisphosphonates [7]. Thus, any intervention that can boost the efficacy of PTH 1-34 could make it more cost-effective. Furthermore, as the length of PTH 1-34 treatment is limited (licensed for up to 18-24 months only), and maximizing its response by such augmentation would be desirable, in terms of reducing fracture risk in individual patients and by potential cost-saving implications.

Mechanical loading is also known to increase bone formation and load-bearing exercise is an important component in maintaining and improving bone health. Studies have shown that load-bearing exercise can increase bone mineral density (BMD) [8].

A synergistic or additive effect of combined PTH 1-34 treatment and mechanical loading has been reported [9, 10]. Not all older people, however, can undertake high-intensity or weight-bearing exercise. A proposed alternative is whole-body vibration (WBV) therapy which, like weight-bearing exercise, stimulates muscles and bones. In some studies, WBV increases the anabolic (bone-building) effects in bone tissue, as well as increasing $\operatorname{BMD}[11,12]$. One hypothesis suggests that the effects of vibration directly activate mechanosensors in bone cells [13]. Like weight-bearing exercise [14], WBV may thus improve muscle strength and power by increasing neuromuscular activation [15]. In healthy volunteers, the effects of vibration therapy on muscle strength and performance were similar to those of short-term resistance exercise [14].

WBV therapy may also improve blood circulation in muscle and bone and increase the supply of nutrients needed to build bones [16]. Platforms for WBV are now commercially available and are used in gyms, as an alternative form of exercise. Animal studies with the combination of PTH analogs and WBV are few and with diverse results. One study with low-magnitude vibration and PTH 1-34 in mice showed no synergy in increasing bone mineral content after 8 weeks of treatment [17]. A recently published study showed that the combination of noise-like WBV and PTH treatment of fracture healing produced an additive effect in increasing bone formation and enhancing the mechanical function of the bone [18]. Human studies have shown that vibration can be anabolic to bone $[12,19,20]$, and a study performed by one of our group (TM) has shown that WBV is well tolerated even by frail, older individuals and increases muscle strength and boneformation markers [19]. Adding mechanical loading in the form of WBV to the treatment with an anabolic agent may improve treatment outcomes further increasing BMD and reducing the risk of fracture.

\section{Study objectives}

To determine if WBV in addition to standard PTH 1-34 treatment has a greater effect in osteoporotic patients compared to standard PTH 1-34 treatment alone on:

\section{BMD}

Bone microarchitecture

Markers of bone formation and resorption

Muscle function and balance

To assess the safety and adherence to WBV in

osteoporotic patients 


\section{Methods/design}

\section{General design}

This will be a multicenter, assessor-blinded, superiority, two-armed RCT in osteoporotic patients starting standard PTH 1-34 treatment. In Denmark, PTH $1-34$ is used for the treatment of severe osteoporosis, and the cost is reimbursed if patients have a lumbar spine or total hip T-score $\leq-3$ combined with at least one clinical vertebral fracture (compression $\geq 25 \%$ ) within the past 3 years or at least two vertebral fractures (compression $\geq 25 \%$ ) independently of BMD. Participants will be randomized to PTH 1-34 treatment alone or to combined PTH 134 treatment and WBV (see Fig. 1). The study will be reported according to the Consolidated Standards of Reporting Trials (CONSORT) extension to nonpharmaceutical interventions and the Standard Protocol Items: Recommendations for Interventional
Trials (SPIRIT) 2013 Checklist for RCTs (Additional file 1).

\section{Participants}

Postmenopausal women over the age of 50 years attending either of the following outpatient clinics will be recruited: the Osteoporosis Clinics at Odense University Hospital (Svendborg or Odense), the Department of Geriatrics at Odense University Hospital, the Department of Endocrinology at Hospital of Southwest Denmark, the Department of Endocrinology at Hospital Lillebaelt or the Department of Endocrinology and Internal Medicine, Aarhus University Hospital.

\section{Inclusion criteria}

Postmenopausal women aged $\geq 50$ years starting PTH 1-34 treatment for osteoporosis

\begin{tabular}{|c|c|c|c|c|c|c|c|c|}
\hline \multirow[b]{3}{*}{ TIMEPOINT** } & \multicolumn{8}{|c|}{ STUDY PERIOD } \\
\hline & \multirow{2}{*}{$\frac{\text { Enrolment }}{-t_{1}}$} & \multirow{2}{*}{$\begin{array}{c}\text { Allocation } \\
0\end{array}$} & \multicolumn{5}{|c|}{ Post-allocation } & \multirow{2}{*}{$\frac{\text { Close-out }}{24}$} \\
\hline & & & 3 & 6 & 12 & 18 & 24 & \\
\hline \multicolumn{9}{|l|}{ ENROLMENT: } \\
\hline \multirow{3}{*}{$\begin{array}{l}\text { Eligibility screen } \\
\text { Informed consent } \\
\text { Demographic data }\end{array}$} & $X$ & & & & & & & \\
\hline & $X$ & & & & & & & \\
\hline & $\mathrm{X}$ & & & & & & & \\
\hline Allocation & & $x$ & & & & & & \\
\hline \multicolumn{9}{|l|}{ INTERVENTIONS: } \\
\hline \multicolumn{9}{|l|}{$\begin{array}{l}\text { PTH }(1-34) \\
\text { treatment }\end{array}$} \\
\hline \multicolumn{9}{|l|}{$\begin{array}{l}\text { Whole-body } \\
\text { vibration }\end{array}$} \\
\hline \multicolumn{9}{|l|}{$\begin{array}{l}\text { Whole-body } \\
\text { vibration }\end{array}$} \\
\hline \multicolumn{9}{|l|}{ ASSESSMENTS: } \\
\hline \multirow{2}{*}{$\begin{array}{l}B M D \\
H R-p Q C T\end{array}$} & & $X$ & & & $X$ & & X & $X$ \\
\hline & & $\mathrm{X}$ & & $X$ & $\mathrm{X}$ & $\mathrm{X}$ & 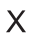 & $X$ \\
\hline \multirow{3}{*}{$\begin{array}{l}\text { Bone biomarkers } \\
\text { Functional } \\
\text { biomarkers }\end{array}$} & & $X$ & $\mathrm{X}$ & $\mathrm{X}$ & $\mathrm{X}$ & $\mathrm{X}$ & $X$ & $X$ \\
\hline & & $X$ & $X$ & $x$ & $X$ & $X$ & $X$ & $x$ \\
\hline & & $X$ & & & $X$ & & X & \\
\hline
\end{tabular}

Fig. 1 The Standard Protocol Items: Recommendations for Interventional Trials (SPIRIT) Figure with the schedule of enrollment, interventions, and assessments. BMD bone mineral density; Bone turnover biomarkers procollagen type-1 amino-terminal propeptide, carboxy-terminal type-1 collagen crosslinks, sclerostin; HR-pQCT high-resolution peripheral quantitative computed tomography; FES-I Falls Efficacy Scale International; Functional biomarkers: Short Physical Performance Battery, Timed Up and Go, grip strength, leg extension power; IPAQ International Physical Activity Questionnaire. 


\section{Exclusion criteria}

Currently taking oral glucocorticoids

Unable to give informed consent

Unable to stand for $1 \mathrm{~min}$ at a time on the vibration

platform

Contraindications to WBV (e.g., joint prosthesis, pacemakers)

\section{Intervention}

After instructions by nurses in the treating clinics all participants will self-administer subcutaneously (s.c) PTH 1-34 (20 $\mu$ g s.c. daily) during the study. The participants follow each clinic's control program including measurements of serum calcium and creatinine throughout the study. Medical records will be used to check the adherence to the PTH 1-34 treatment.

Half of the participants will be randomized to an intervention group, receiving WBW as add-on treatment. WBV will be undertaken using power plate My5 (Power plate, UK). The power plate machine oscillates in all three planes, with a frequency of $30 \mathrm{~Hz}$ and amplitude of $1 \mathrm{~mm}$ (low displacement) and peak acceleration of $35.53 \mathrm{~ms}^{-2} \mathrm{rms}(3.6 \mathrm{~g})$.

The WBV intervention will be performed three times a week according to a training protocol (Table 1). The vibration platforms will be delivered and installed in the participant's own home and instructions for use and the training program will be given by one of the investigators (DJ).

The WBV intervention will be conducted with the knees slightly bent (at approximately $20^{\circ}$ ) to prevent vibrations to cause side effect as dizziness from the vibration transmitted to the head. The training period will be followed by a resting period in the ratio $1: 1$ and the training days will be conducted with a resting day in between.

\section{Randomization}

After informed consent and collection of baseline data, participants will be randomized into two groups: PTH 1-34 treatment alone or PTH 1-34 treatment + WBV (Fig. 2). The randomization is a web-based, computer-generated block randomization with no stratification. Block size is created by a data manager and the size is unknown to the investigators until the end of the study. Allocation will be concealed to the participants and the investigators until after collection of baseline data.

After 12 months the combined PTH 1-34 + WBV group will be re-randomized to continuation of combined PTH 1-34 + WBV for another 12 months, or to PTH 1-34 alone (i.e., stopping WBV) for another 12 months. This will allow us to determine if any positive effects of WBV wear off after it has been stopped or if any gains persist.

\section{Endpoints}

\section{Primary endpoints}

The primary endpoint is the percent change in areal BMD of total hip and lumbar spine as measured by dual-energy x-ray absorptiometry (DXA) (Hologic Discovery, Waltham, MA, USA) from baseline until 12 months (Table 2).

BMD is chosen as an endpoint since it is an important determinant of fracture risk and is used to diagnose and assess response to treatment in osteoporotic patients $[1,21]$.

\section{Secondary endpoints}

Bone mass Change in areal BMD of total hip and lumbar spine is measured by DXA from baseline until 6, 18, and 24 months (Table 2).

Bone microarchitecture Images from high-resolution peripheral quantitative computed tomography (HRpQCT) (Xtreme CT, Scanco Medical, AG, Brüttisellen, Switzerland), of the non-dominant distal radius and distal tibia (the opposite limb in the presence of a previous fracture) will be obtained to measure bone geometry, cortical morphology, trabecular morphology, and overall biomechanical competence. In in-vitro studies estimated bone strength of the radius using finite element analysis $[22,23]$ which has been shown to be more closely correlated to observed radius bone strength than areal BMD by DXA [22]. PTH 1-34 treatment has been shown to be associated with an increase in vertebral estimated bone strength [24] and preservation of the estimated bone strength of the radius and hip $[25,26]$. The images are

Table 1 Training protocol

\begin{tabular}{llll}
\hline Week & First training day & Second training day & Third training day \\
\hline 1 & $30 \mathrm{~s} \times 2$ & $30 \mathrm{~s} \times 3$ & $30 \mathrm{~s} \times 4$ \\
2 & $1 \min \times 1$ & $1 \min \times 2$ & $1 \mathrm{~min} \times 3$ \\
$3-5$ & $1 \min \times 4$ & $1 \min \times 4$ & $1 \mathrm{~min} \times 4$ \\
$6-7$ & $1 \min \times 5$ & $1 \min \times 5$ & $1 \mathrm{~min} \times 5$ \\
8 to the end of study & $1 \min \times 6$ & $1 \min \times 6$ & $1 \mathrm{~min} \times 6$ \\
\hline
\end{tabular}

Min minutes, $s$ seconds 


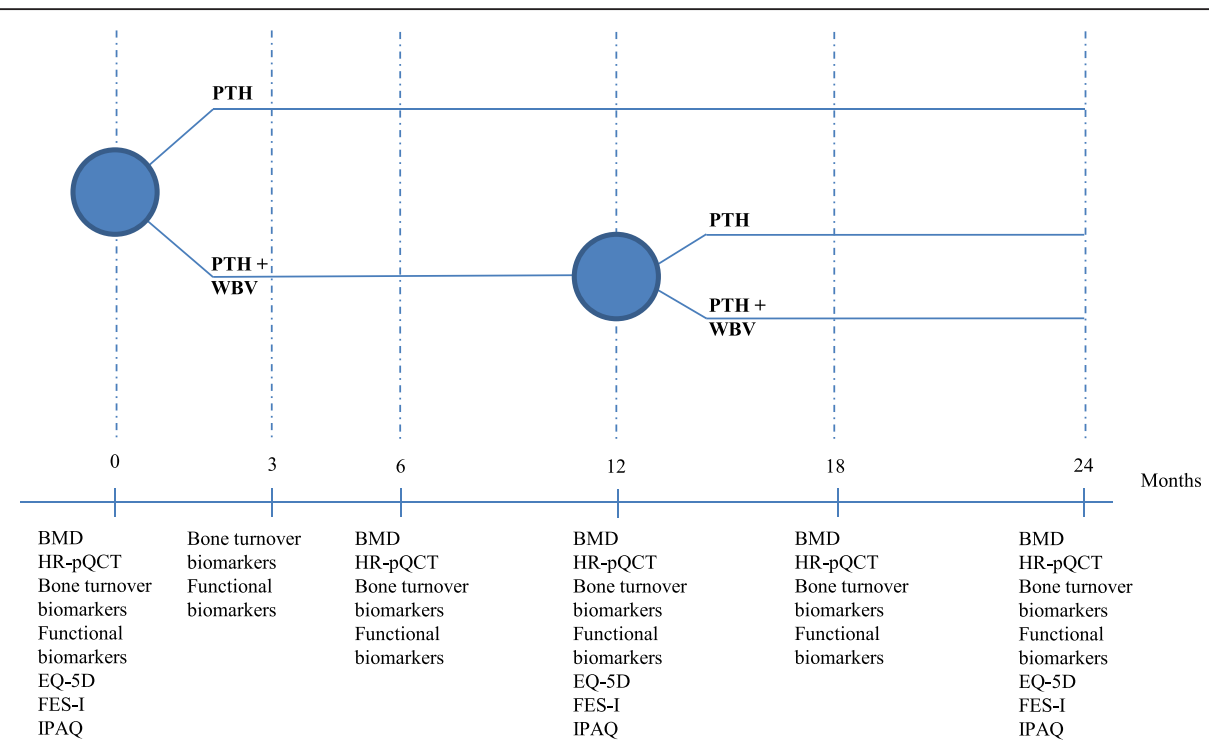

Fig. 2 Timeline for participants. Figure 2 shows the timeline for the participants in the study. The $x$-axis is the time during the study in months. The participants will be randomized after baseline data is collected to an intervention group receiving whole-body vibration (WBV) in addition to PTH 1-34 compared to the control group receiving PTH 1-34 alone. After 12 months the intervention group will be re-randomized. BMD bone mineral density; HR-pQCT high-resolution peripheral quantitative computed tomography; Bone biomarkers procollagen type-1 amino-terminal propeptide (P1NP), carboxy-terminal type-1 collagen crosslinks (CTX), and sclerostin; Physical markers: Timed Up and Go (TUG), Short Physical Performance Battery (SPPB), grip strength and leg extensor power; DXA dual-energy x-ray absorptiometry; IPAQ The International Physical Activity Questionnaire; FES-I Falls Efficacy Scale International

Table 2 Primary and secondary endpoints

\begin{tabular}{|c|c|c|c|}
\hline & Measurement & Site & Time (months) \\
\hline \multicolumn{4}{|l|}{ Primary endpoints } \\
\hline$\Delta$ Bone mineral density & DXA & $\begin{array}{l}\text { Total hip } \\
\text { Spinal region }\end{array}$ & 12 \\
\hline \multicolumn{4}{|l|}{ Secondary endpoints } \\
\hline$\Delta$ Bone mineral density & DXA & $\begin{array}{l}\text { Total hip } \\
\text { Spinal region }\end{array}$ & 6,18, and 24 \\
\hline$\Delta$ Bone microarchitecture & HR-pQCT & $\begin{array}{l}\text { Distal radius } \\
\text { Distal tibia }\end{array}$ & $6,12,18$, and 24 \\
\hline$\Delta$ Bone biomarkers & $\begin{array}{l}\text { P1NP } \\
\text { CTX, sclerostin }\end{array}$ & Serum & $3,6,12,18$, and 24 \\
\hline \multirow[t]{3}{*}{$\Delta$ Physical biomarkers } & $\begin{array}{l}\text { TUG } \\
\text { SPPB }\end{array}$ & & $3,6,12,18$, and 24 \\
\hline & Dynamometer & Hand grip & \\
\hline & Nottingham power rig & Leg extensor & \\
\hline$\Delta$ Quality of life & EQ-5D & & 12 and 24 \\
\hline$\Delta$ Fear of falling & FES-I & & 12 and 24 \\
\hline Physical activity & IPAQ & & 0,12 , and 24 \\
\hline Adherence to WBV & Training logbook & & Continuously \\
\hline Falls & Falls calendar & & Continuously \\
\hline
\end{tabular}

$\triangle$ change from baseline; Time is measured in months; DXA dual-energy x-ray absorptiometry; HR-pQCT high-resolution peripheral quantitative computed tomography; P1NP procollagen type-1 amino-terminal propeptide; CTX carboxy-terminal type-1 collagen crosslinks; TUG Timed Up and Go; SPPB Short Physical Performance Battery; FES-I Falls Efficacy Scale International; IPAQ The International Physical Activity Questionnaire; EQ-5D EuroQoL 5-dimension, 
obtained at baseline, $6,12,18$, and 24 months (Table 2). The reported outcomes will be total BMD, cortical thickness, cortical porosity, bone volume per trabecular volume (BV/TV), trabecular number, trabecular thickness, and finite element failure load. The scanning protocol and the image acquisition have previously been described in detail [22, 23].

Serum bone turnover markers Markers of bone formation (procollagen type-1 amino-terminal propeptide (P1NP)) and bone resorption (carboxy-terminal type-1 collagen crosslinks (CTX-1) will be measured by the method of chemiluminescence (iSYS, Immunodiagnostic Systems Ltd., Boldon, England). Sclerostin is analyzed using TECOmedical Human Sclerostin HS ELISA (TECOmedical group, Sissach, Switzerland). Bone turnover is affected by treatment with PTH 1-34, and studies have shown that a rise in the marker of bone formation, P1NP, during PTH 1-34 treatment is a predictor of the increase in BMD [27]. The assessment of bone biomarkers will allow exploration of mechanisms of action of WBV in augmenting PTH 1-34 treatment on bone turnover. The blood samples are taken at baseline, 3, 6, 12,18 , and 24 months (Table 2) after an overnight fast and stored at $-70{ }^{\circ} \mathrm{C}$ until analyzed in a central laboratory.

Functional biomarkers Functional biomarkers including measurements of muscle strength, function and balance is measured by: (1) Timed Up and Go (TUG), (2) Short Physical Performance Battery (SPPB), (3) leg extensor power (Nottingham power rig), and (4) grip strength (Smedley dynamometer, TTM, Tokyo, Japan).

Fragility fractures are most often associated with falls $[21,28]$. The measurement of lower extremity function and mobility will indicate if WBV has the potential to reduce the risk of falls in osteoporotic patients, which is another important determinant of the risk of fracture, independent on the effects on bone [21]. The TUG is the measurement of functional mobility and is a simple and reliable clinical test used to screen for fall risk in community-dwelling older persons [29]. Lowerextremity function and balance can also be assessed by the SPPB which is a validated test in older adults [30].

Leg extension power is assessed using a leg extensor power rig, (Medical Engineering Unit, School of Biomedical Sciences, University of Nottingham Medical School, Nottingham, UK) reported to have good reliability with no significant differences found between two tests performed 1 week apart $(r=0.97$; coefficient of variation $=9 \%$ ) [31]. At each visit the same leg is tested with a series of five measurements from the same starting position. The leg is selected randomly at the first visit. The SPPB and the TUG are described in detail by others [30, 32]. Reduced grip strength is a predictor of fragility fractures independent of BMD [33]. A reliable and valid method is by using a Smedley dynamometer (TTM, Tokyo, Japan). The measurements are done twice with each hand using a protocol described by others [34].

The functional tests will be performed by technicians, who all have been trained by a physiotherapist and all tests are conducted by standardized protocols. The TUG, SPPB, grip strength and leg extension power tests will be performed at baseline, 3, 6, 12, 18, and 24 months (Table 2).

Full-body DXA (Hologic Discovery, Waltham, MA, USA) is performed at baseline, 12 , and 24 months to assess muscle mass (Fig. 2).

Sarcopenia is a condition with low skeletal muscle mass leading to decreased muscle strength, and impaired physical function, associated with an increased risk of falls [35]. Sarcopenia is proposed to be assessed by a variety of measurements of muscle mass and function including but not limited to full-body DXA, SPPB, grip strength, and gait speed [36, 37].

Questionnaires The participants' physical activity will be measured by a validated questionnaire, the International Physical Activity Questionnaire (IPAQ). The participants' multiples of the resting metabolic rate will be generated (MET-min/week) [38].

Vertebral fractures have been shown to decrease quality of life [3] and the EuroQol 5-dimension, 5-level questionnaire (EQ-5D) is a well-validated measure of quality of life [39, 40].

The patients' own fear of falling is associated with the risk of falls [41] and the the Falls Efficacy Scale International (FES-I) questionnaire, which is a reliable and validated questionnaire to measure the fear of falling in an older population [42].

The questionnaires IPAQ, EQ-5D, and FES-I will be distributed in paper versions to the participants at baseline, 12 , and 24 months.

Adherence Adherence to WBV is ascertained using self-reported adherence in a log book. Dropouts and the reasons for this will be noted. The adherence to the PTH 1-34 treatment is ascertained by asking the patients and by using the prescription database.

Side effects Information on symptoms of dizziness, pain, and falls will be collected. The participants will be handed a calendar to note fall events, defining a fall as "an unexpected event in which the participants come to rest on the ground, floor, or lower level" [43]. Pain will be assessed by the Numeric Rank Score (0 being no pain, and 10 being unbearable pain) and the question about how many days they have felt dizzy during the last 
week, and falls will be assessed by monthly telephone calls during the first year and then every 3 months for the rest of the trial.

Demographic data, including data on risks factors associated with osteoporosis and fragility factures, will be collected at baseline and thus before randomization.

These factors include Body Mass Index, current and previous height, age, comorbidity, fall history, previous fractures, vitamin $\mathrm{D}$ and calcium intake, current, and previous history of medication.

\section{Statistical analysis Sample size determination}

The inclusion of 32 participants (16 in each group) would give the study $80 \%$ power to detect a clinically significant additional increase of $22 \%$ in BMD with WBV (assuming a 9\% increase of BMD in the PTH 134-alone group and $11 \%$ increase in the combined PTH 1-34 + WBV group, and assuming a standard deviation (SD) of the BMD increase of $2 \%$ [12]. Inclusion of 40 participants (20 in each group) will allow for a $20 \%$ dropout rate.

\section{Statistical methods and data managing}

The intervention arm (PTH + WBV) is compared against the control arm for all primary analyses. We will use analysis of covariance (ANCOVA) and "percentage change" for primary outcome adjusting for baseline measurements. For secondary outcomes we will use a chisquared test for binary outcomes, analysis of variance (ANOVA) and the $T$ test for continuous outcomes, and for non-normally distributed continuous outcomes we will use log transformation or Wilcoxon's match-pairs signed-rank test and the Kruskal-Wallis test.

All $p$ values will be reported to four decimal places with $p$ values $<0.001$ reported as $p<0.001$. STATA version 14 will be used to conduct analyses. For all tests, we will use two-sided $p$ values with an alpha $\leq 0.05$ level of significance.

The analyses will be conducted with the intention-totreat method, and in order to investigate the effect of adherence and withdrawal a per-protocol analysis will be conducted. Missing data will be handled using multiple imputation. Study data is collected and managed using REDCap (Version 6.5.10 - @ 2015 Vanderbilt University) electronic data capture tools hosted at the University of Southern Denmark [44]. Sequential study numbers will be allocated to study patients and entered into the recruitment log at the secure web database REDCap.

\section{Discussion}

This is a RCT in osteoporotic patients randomized to PTH 1-34 treatment alone or to combined PTH 1-34 treatment and WBV. The primary and secondary endpoints in the study are chosen because previous studies have shown such markers of bone mass, microarchitecture, and turnover, as well as indices of physical performance, to be associated with osteoporosis or fragility fractures.

Earlier studies have reported WBV as a feasible intervention [12] and in older patients the adherence is reported to be good [19].

The intervention is designed to be of long duration (12-24 months) and the setting at the participants' own home makes the training easy and highly accessible.

The study has limitations including the non-blinded design. It is our hypothesis that the primary endpoint, the BMD as well as a number of secondary endpoints (bone microarchitecture and bone turnover markers) are not at risk of bias by the open-label design. The functional biomarkers on the other hand could be affected by the non-blinded design but the study personnel performing the tests are blinded to the allocation and the baseline data is collected before randomization.

Measurements of quality of life, physical activity, and the FES-I questionnaire are patient-collected data, resulting in the possibility of bias in the reporting.

To limit the possibility of bias by attention, the participants will receive close to an equal amount of contacts by the investigators because of the "at home training design.".

The study is partially blinded and is protected against selection bias by central randomization after baseline measurements, randomization via web with no stratification, and by allocation concealment.

It is our hypothesis that the DXA, HR-pQCT, and bone marker measurements are not at risk of performance bias. The physical biomarkers may be prone to performance bias, but the test personnel will be blinded to avoid evaluation bias.

To avoid attrition in both arms and ensure adherence, the participants are contacted by telephone each month during the first year and every third month during the second year. The participants receive a direct telephone number and mailing address to the primary investigator and are encouraged to make contact in case of any questions or events.

Every patient is motivated to attend follow-up regardless of randomization or adherence. The participants do not receive payment but are reimbursed for transport expenses.

The adherence to the training protocol is patient reported data and is in risk of reportingbias. A previous study nevertheless found high correlation with the usage of a training logbook to electronic monitoring in the WBV intervention in older adults (overall intraclass correlation coefficient $=0.96$ ), showing a small risk of bias in the collected data on adherence [45]. 
A combined treatment of WBV and PTH 1-34 might have synergistic or additive beneficial effects on bone strength, thereby reducing fracture risk and making the treatment more cost-effective. A beneficial effect of WBV on muscles, and subsequently fall risk, may lower the fracture risk even further, resulting in comprehensive fracture prevention.

\section{Trial status}

Recruitment of participants started in November 2015 and will be completed in September 2017. This study has been registered at ClinicalTrials.gov (September, 2015: NCT02563353).

\section{Additional file}

Additional file 1: SPIRIT 2013 Checklist: recommended items to address in a clinical trial protocol and related documents*. (DOC $124 \mathrm{~kb}$ )

\begin{abstract}
Abbreviations
BMD: Bone mineral density; CTX: Carboxy-terminal type-1 collagen crosslinks; DXA: Dual-energy x-ray absorptiometry; FES-I: Falls Efficacy Scale International; HR-pQCT: High-resolution peripheral quantitative computed tomography; IPAQ: International Physical Activity Questionnaire; P1NP: Procollagen type-1 amino-terminal propeptide; PTH 1-34: Teriparatide; RCT: Randomized controlled trial; SPPB: Short Physical Performance Battery; TUG: Timed Up and Go; WBV: Whole-body vibration
\end{abstract}

\section{Acknowledgements}

The team would like to thank Lars Erik Matzen for advice and support during the study.

\section{Funding}

The study is funded by The Odense University Hospital Research Foundation, the Region of Southern Denmark PhD Foundation, The Research Foundation between Odense University Hospital and Rigshospitalet, OPEN - Odense Patient data Explorative Network, The A.P. Møller Foundation for the Advancement of Medical Science, and the Danish Osteoporosis Foundation. The biobank and data management is conducted in collaboration with OPEN - Odense Patient data Explorative Network.

The funding parties have no influence on the study design, study conduct, results, or dissemination.

\section{Availability of data and materials}

The trial dataset is accessed by the investigators (DJ, JR, TM). DJ takes responsibility for the integrity of the dataset. There are no results available from this study protocol. The public can access the data by request.

\section{Authors' contributions}

JR and TM: idea conception, original protocol, statistical plan, sample size, and design. DJ, JR, NJ, SH, and TM: endpoint design. DJ and JR: trial management, protocol amendments, updates, and protocol paper draft. DJ, JR, NJ, SH, and TM: writing and editing. All authors reviewed and commented on drafts of the paper and approved the final version.

\section{Ethics approval and consent to participate}

This study will be conducted according to the standards of International Conference on Harmonization, Research Ethics Committee regulations, any applicable government regulations (e.g., The Danish Data Protection Agency), and local procedures. The protocol is approved by the Regional Scientific Ethical Committee of Southern Denmark (ref. ID S-20150121) and The Danish Data Protection Agency (2008-58-0035, 13/40496). Any amendments will be submitted to the above ethical committee for approval of the study conduct. The participants are covered by the Danish "Complaint and compensation law in health care." Written informed consent will be obtained from each participant. The trial is audited in an ongoing manner to insure patient safety. All events and adverse events are audited each year. In case of serious adverse events these will be sent for audit within 2 weeks. The events are audited independent from the trial investigators by The Regional Scientific Ethical Committee of Southern Denmark.

\section{Consent for publication}

Not applicable

\section{Competing interests}

The investigators have no competing interests in the study.

\section{Publisher's Note}

Springer Nature remains neutral with regard to jurisdictional claims in published maps and institutional affiliations.

\section{Author details}

'Department of Geriatric Medicine, Odense University Hospital, Odense, Denmark. ${ }^{2}$ Institute of Clinical Research, Faculty of Health Science, University of Southern Denmark, Odense, Denmark. ${ }^{3}$ Department of Clinical Biochemistry, Rigshospitalet, Copenhagen, Denmark. ${ }^{4}$ OPEN - Odense Patient data Explorative Network, The University of Southern Denmark, Odense, Denmark. ${ }^{5}$ Department of Endocrinology, Odense University Hospital, Odense, Denmark. ${ }^{6}$ Department of Geriatric Medicine, Nottingham University Hospitals Trust NHS, Nottingham, UK.

Received: 3 May 2017 Accepted: 16 February 2018

Published online: 16 March 2018

\section{References}

1. World Health Organization. Assessment of fracture risk and its application to screening for postmenopausal osteoporosis. WHO technical report series. Geneva: WHO; 1994

2. Ryg J, Rejnmark L, Overgaard S, Brixen K, Vestergaard P. Hip fracture patients at risk of second hip fracture: a nationwide population-based cohort study of 169,145 cases during 1977-2001. J Bone Miner Res Off J Am Soc Bone Miner Res. 2009;24(7):1299-307.

3. Borgstrom F, Zethraeus N, Johnell O, Lidgren L, Ponzer S, Svensson O, et al. Costs and quality of life associated with osteoporosis-related fractures in Sweden. Osteoporos Int. 2006;17(5):637-50.

4. Johnell O, Kanis JA. An estimate of the worldwide prevalence and disability associated with osteoporotic fractures. Osteoporos Int. 2006:17(12):1726-33.

5. Hansen L, Mathiesen AS, Vestergaard P, Ehlers LH, Petersen KD. A health economic analysis of osteoporotic fractures: who carries the burden? Arch Osteoporos. 2013:8:126.

6. Neer RM, Arnaud CD, Zanchetta JR, Prince R, Gaich GA, Reginster J-Y, et al. Effect of parathyroid hormone (1-34) on fractures and bone mineral density in postmenopausal women with osteoporosis. N Engl J Med. 2001;344(19): 1434-41.

7. Blick SK, Dhillon S, Keam SJ. Teriparatide: a review of its use in osteoporosis. Drugs. 2008;68(18):2709-37.

8. Rizzoli R, Bianchi ML, Garabedian M, McKay HA, Moreno LA. Maximizing bone mineral mass gain during growth for the prevention of fractures in the adolescents and the elderly. Bone. 2010;46(2):294-305.

9. Roberts MD, Santner TJ, Hart RT. Local bone formation due to combined mechanical loading and intermittent hPTH-(1-34) treatment and its correlation to mechanical signal distributions. J Biomech. 2009:42(15):2431-8.

10. Sugiyama T, Saxon LK, Zaman G, Moustafa A, Sunters A, Price JS, et al. Mechanical loading enhances the anabolic effects of intermittent parathyroid hormone (1-34) on trabecular and cortical bone in mice. Bone. 2008;43(2):238-48

11. Gusi N, Raimundo A, Leal A. Low-frequency vibratory exercise reduces the risk of bone fracture more than walking: a randomized controlled trial. BMC Musculoskelet Disord. 2006:7:92.

12. Verschueren SM, Roelants M, Delecluse C, Swinnen S, Vanderschueren D, Boonen S. Effect of 6-month whole body vibration training on hip density, muscle strength, and postural control in postmenopausal women: a randomized controlled pilot study. J Bone Miner Res Off J Am Soc Bone Miner Res. 2004;19(3):352-9. 
13. Rubin $\mathrm{C}, \mathrm{Xu} \mathrm{G}$, Judex $\mathrm{S}$. The anabolic activity of bone tissue, suppressed by disuse, is normalized by brief exposure to extremely low-magnitude mechanical stimuli. FASEB J. 2001;15(12):2225-9.

14. Erskine J, Smillie I, Leiper J, Ball D, Cardinale M. Neuromuscular and hormonal responses to a single session of whole body vibration exercise in healthy young men. Clin Physiol Funct Imaging. 2007;27(4):242-8.

15. Pollock RD, Woledge RC, Mills KR, Martin FC, Newham DJ. Muscle activity and acceleration during whole body vibration: effect of frequency and amplitude. Clin Biomech (Bristol, Avon). 2010;25(8):840-6.

16. Kerschan-Schindl K, Grampp S, Henk C, Resch H, Preisinger E, Fialka-Moser V, et al. Whole-body vibration exercise leads to alterations in muscle blood volume. Clin Physiol. 2001;21(3):377-82.

17. Lynch MA, Brodt MD, Stephens AL, Civitelli R, Silva MJ. Low-magnitude whole-body vibration does not enhance the anabolic skeletal effects of intermittent PTH in adult mice. J Orthop Res. 2011:29(4):465-72.

18. Matsumoto T, Sato D, Hashimoto $Y$. Individual and combined effects of noise-like whole-body vibration and parathyroid hormone treatment on bone defect repair in ovariectomized mice. Proc Inst Mech Eng H J Eng Med. 2016;230(1):30-8.

19. Corrie H, Brooke-Wavell K, Mansfield NJ, Cowley A, Morris R, Masud T. Effects of vertical and side-alternating vibration training on fall risk factors and bone turnover in older people at risk of falls. Age Ageing. 2015;44(1):115-22.

20. Gilsanz V, Wren TA, Sanchez M, Dorey F, Judex S, Rubin C. Low-level, highfrequency mechanical signals enhance musculoskeletal development of young women with low BMD. J Bone Miner Res Off J Am Soc Bone Miner Res. 2006;21(9):1464-74.

21. Cummings SR, Nevitt MC, Browner WS, Stone K, Fox KM, Ensrud KE, et al. Risk factors for hip fracture in white women. Study of osteoporotic fractures research group. N Engl J Med. 1995;332(12):767-73.

22. Pistoia W, van Rietbergen B, Lochmüller EM, Lill CA, Eckstein F, Rüegsegge $P$. Image-based micro-finite-element modeling for improved distal radius strength diagnosis: moving from "bench" to "bedside". J Clin Densitom. 2004;7(2):153-60.

23. Boutroy S, Bouxsein ML, Munoz F, Delmas PD. In vivo assessment of trabecular bone microarchitecture by high-resolution peripheral quantitative computed tomography. J Clin Endocrinol Metab. 2005;90(12):6508-15.

24. Graeff C, Chevalier Y, Charlebois M, Varga P, Pahr D, Nickelsen TN, et al. Improvements in vertebral body strength under teriparatide treatment assessed in vivo by finite element analysis: results from the EUROFORS study. J Bone Miner Res Off J Am Soc Bone Miner Res. 2009;24(10):1672-80.

25. Hansen S, Hauge EM, Beck Jensen JE, Brixen K. Differing effects of PTH 1-34, PTH 1-84, and zoledronic acid on bone microarchitecture and estimated strength in postmenopausal women with osteoporosis: an 18-month openlabeled observational study using HR-pQCT. J Bone Miner Res Off J Am Soc Bone Miner Res. 2013;28(4):736-45.

26. Keaveny TM, McClung MR, Wan X, Kopperdahl DL, Mitlak BH, Krohn K. Femoral strength in osteoporotic women treated with teriparatide or alendronate. Bone. 2012;50(1):165-70.

27. Bauer DC, Garnero P, Bilezikian JP, Greenspan SL, Ensrud KE, Rosen CJ, et al. Short-term changes in bone turnover markers and bone mineral density response to parathyroid hormone in postmenopausal women with osteoporosis. J Clin Endocrinol Metab. 2006;91(4):1370-5.

28. Parkkari J, Kannus P, Palvanen M, Natri A, Vainio J, Aho H, et al. Majority of hip fractures occur as a result of a fall and impact on the greater trochanter of the femur: a prospective controlled hip fracture study with 206 consecutive patients. Calcif Tissue Int. 1999;65(3):183-7.

29. Shumway-Cook A, Brauer S, Woollacott M. Predicting the probability for falls in community-dwelling older adults using the Timed Up \& Go test. Phys Ther. 2000:80(9):896-903.

30. Freire AN, Guerra RO, Alvarado B, Guralnik JM, Zunzunegui MV. Validity and reliability of the short physical performance battery in two diverse older adult populations in Quebec and Brazil. J Aging Health. 2012;24(5):863-78.

31. Bassey EJ, Short AH. A new method for measuring power output in a single leg extension: feasibility, reliability and validity. Eur J Appl Physiol Occup Physiol. 1990;60(5):385-90.

32. Bohannon RW. Reference values for the Timed Up and Go test: a descriptive meta-analysis. J Geriatr Phys Ther. 2006;29(2):64-8.

33. Albrand G, Munoz F, Sornay-Rendu E, DuBoeuf F, Delmas PD. Independent predictors of all osteoporosis-related fractures in healthy postmenopausal women: the OFELY study. Bone. 2003:32(1):78-85.
34. Frederiksen H, Hjelmborg J, Mortensen J, McGue M, Vaupel JW, Christensen K. Age trajectories of grip strength: cross-sectional and longitudinal data among 8,342 Danes aged 46 to 102. Ann Epidemiol. 2006;16(7):554-62.

35. Bischoff-Ferrari HA, Orav JE, Kanis JA, Rizzoli R, Schlogl M, Staehelin HB, et al. Comparative performance of current definitions of sarcopenia against the prospective incidence of falls among community-dwelling seniors age 65 and older. Osteoporos Int. 2015;26(12):2793-802.

36. Cooper C, Dere W, Evans W, Kanis JA, Rizzoli R, Sayer AA, et al. Frailty and sarcopenia: definitions and outcome parameters. Osteoporos Int. 2012;23(7): 1839-48.

37. Cruz-Jentoft AJ, Baeyens JP, Bauer JM, Boirie Y, Cederholm T, Landi F, et al. Sarcopenia: European consensus on definition and diagnosis: report of the European working group on sarcopenia in older people. Age Ageing. 2010; 39(4):412-23.

38. Ekelund U, Sepp H, Brage S, Becker W, Jakes R, Hennings M, et al. Criterionrelated validity of the last 7-day, short form of the international physical activity questionnaire in Swedish adults. Public Health Nutr. 2006;9(2):258-65.

39. EuroQol group. EuroQol-a new facility for the measurement of healthrelated quality of life. Health Policy. 1990;16(3):199-208.

40. Wittrup-Jensen KU, Lauridsen J, Gudex C, Pedersen KM. Generation of a Danish TTO value set for EQ-5D health states. Scand J Public Health. 2009; 37(5):459-66.

41. Friedman SM, Munoz B, West SK, Rubin GS, Fried LP. Falls and fear of falling: which comes first? A longitudinal prediction model suggests strategies for primary and secondary prevention. J Am Geriatr Soc. 2002;50(8):1329-35.

42. Delbaere K, Close JC, Mikolaizak AS, Sachdev PS, Brodaty H, Lord SR. The Falls Efficacy Scale International (FES-I). A comprehensive longitudinal validation study. Age Ageing. 2010;39(2):210-6.

43. Lamb SE, Jorstad-Stein EC, Hauer K, Becker C. Development of a common outcome data set for fall injury prevention trials: the prevention of falls network Europe consensus. J Am Geriatr Soc. 2005:53(9):1618-22.

44. Harris PA, Taylor R, Thielke R, Payne J, Gonzalez N, Conde JG. Research electronic data capture (REDCap) — a metadata-driven methodology and workflow process for providing translational research informatics support. J Biomed Inform. 2009;42(2):377-81.

45. Jeffrey BA, Hannan MT, Quinn EK, Zimmerman S, Barton BA, Rubin CT, et al. Self-reported adherence with the use of a device in a clinical trial as validated by electronic monitors: the VIBES study. BMC Med Res Methodol. 2012;12:171

\section{Submit your next manuscript to BioMed Central and we will help you at every step:}

- We accept pre-submission inquiries

- Our selector tool helps you to find the most relevant journal

- We provide round the clock customer support

- Convenient online submission

- Thorough peer review

- Inclusion in PubMed and all major indexing services

- Maximum visibility for your research

Submit your manuscript at www.biomedcentral.com/submit
C Biomed Central 